\title{
Influencia del ciclo menstrual en nadadoras
}

\author{
Influence of the menstrual cycle on swimmers
}

\author{
Leila Yajaira Iturralde Guerra ${ }^{1}$ \\ Recibido: 14-10-2020 / Revisado:17-11-2020 /Aceptado: 11-12-2020/ Publicado: 02-01-2021
}

Abstract.

DOI: https://doi.org/10.33262/cienciadigital.v5i1.1464

Introduction. The optimization of sports performance presupposes considering several aspects. In swimming, as in other sports, it is necessary to determine to what extent does the menstrual cycle influence the sports performance of swimmers? Objective. Reflect on the influence of the menstrual cycle in optimizing the athletic performance of swimmers. Methodology. A non-experimental descriptive methodology was followed, with the use of theoretical methods, among these: the historical-logical, the analytical synthetic, inductive - deductive and the bibliographic review; All of this allowed for a bibliographic search in a range between 2010 and 2020. Results. Twenty studies were identified as potentially relevant investigations, of which $100 \%$ combined the variables: menstrual cycle and sports performance and 3 were directed at the incidence of the menstrual cycle in swimmers for $15 \%$ of the sources consulted. The analysis of the preceding theories allowed supporting two fundamental ideas: the first establishes the relationship between the menstrual cycle and sports performance, however a single criterion regarding the degree of influence of the menstrual cycle on sports performance has not yet been achieved, the second idea reaffirms the need to deepen the diagnosis and characteristics of each athlete in order to consider the adaptability of the activities and actions to be carried out during their menstrual cycle within the sports training planning process. Conclusion. The trends in the reviewed theories, despite the diversity and existing controversy, aim to establish the relationship between the menstrual cycle and the athletic performance of athletes, with special interest in female swimmers, highlighting that to achieve the optimization of sports performance it is necessary to consider the principle of individualization of training and the adaptability to the characteristics and phases of the menstrual cycle of each swimmer.

\footnotetext{
${ }^{1}$ Universidad Central del Ecuador, Quito Ecuador, lyiturralde@uce.edu.ec ORCID: https://orcid.org/0000-0003-2999-5534
} 
Keywords: menstrual cycle, sports performance, swimmers.

\section{Resumen.}

Introducción. La optimización del rendimiento deportivo presupone considerar varios aspectos. En la natación como en el resto de los deportes es preciso determinar ¿En qué medida influye el ciclo menstrual en el rendimiento deportivo de las nadadoras?, Objetivo. Reflexionar sobre la influencia del ciclo menstrual en la optimización del rendimiento deportivo de las nadadoras. Metodología. Se siguió una metodología descriptiva no experimental, con la utilización de métodos teóricos, dentro de estos: el histórico-lógico, el analítico - sintético, inductivo - deductivo y la revisión bibliográfica; todo ello permitió realizar un rastreo bibliográfico en un rango comprendido entre el 2010 y el 2020. Resultados. Se determinaron como investigaciones potencialmente relevantes 20 estudios, de estos el 100\% maneja de forma combinada las variables: ciclo menstrual y rendimiento deportivo y 3 se dirigen a la incidencia del ciclo menstrual en nadadoras para un $15 \%$ de las fuentes consultadas. El análisis de las teorías precedentes permitió sustentar dos ideas fundamentales: la primera establece la relación entre ciclo menstrual y rendimiento deportivo, sin embargo aún no se logra un criterio único respecto al grado de influencia del ciclo menstrual sobre el rendimiento deportivo, la segunda idea reafirma la necesidad de profundizar en el diagnóstico y características de cada deportista para poder considerar dentro del proceso de planificación del entrenamiento deportivo la adaptabilidad de las actividades y acciones a desarrollar durante su ciclo menstrual. Conclusión. Las tendencias en las teorías revisadas a pesar de la diversidad y controversia existente apuntan a establecer la relación entre el ciclo menstrual y el rendimiento deportivo de las atletas, con especial interés en las nadadoras, resaltan que para lograr la optimización del rendimiento deportivo es necesario considerar el principio de individualización del entrenamiento y su adaptabilidad a las características y fases del ciclo menstrual de cada nadadora.

Palabras claves: ciclo menstrual, rendimiento deportivo, nadadoras.

\section{Introducción.}

El término menstruación proviene del latín menstruum, que a su vez viene de menstruus, que significa mensual. El equivalente griego para la palabra mensual es menos, por lo que algunos términos que se refieren a la menstruación llevan este prefijo: menarquia, menorragia, menometrorragia (Rello, 2010). Indica este autor que según la Real Academia Española de la Lengua (RAE), menstruar se entiende como "Evacuar la sangre procedente de la matriz que todos los meses evacuan naturalmente las mujeres y las hembras de ciertos animales". (pág1).

Como se ha venido sistematizando en los estudios e investigaciones preliminares el desarrollo de las niñas y niños es producto de diversos cambios, tanto biológicos, psicológicos, cognitivos como sociales. Según, Piaget \& Inherder (1948), este largo proceso incluye diferentes etapas o estadios que van conformando desde el nacimiento 
hasta la adultez los períodos y fases evolutivas del desarrollo. Es en este proceso de desarrollo donde se dan los períodos sensibles en las diferentes edades (Sánchez et al., 2020; Betancourt, Beatriz, Arias \& Barroso, 2020).

En el caso de las niñas una etapa fundamental en su vida está relacionada con el inicio del ciclo menstrual y con este la aparición de la primera menstruación. La llegada de la menstruación no solo implica cambios fisiológicos, sino también psicológicos, con los cuales la niña debe aprender a convivir. Prepararla para este momento sigue constituyendo una alta responsabilidad tanto para padres como para el contexto pedagógico y social donde se desenvuelve.

Las Doctoras Allen, \& Miller (2019), en su trabajo sobre la condición física en las niñas, establecen que la pubertad suele comenzar antes de lo que los padres creen, en especial en las niñas. Apuntan que aunque el comienzo puede variar, la mayoría de las niñas tienen su primer período menstrual de 2 a 3 años después del desarrollo de los botones mamarios y con este se suceden múltiples cambios.

Ahora bien, cuando las niñas inician la práctica deportiva desde edades tempranas y se convierte en atletas activas la aparición del ciclo menstrual trae consigo una serie de situaciones que deben ser guiadas y controladas oportunamente, tanto por los entrenadores, directivos, como por los padres de familia. En esta línea de pensamientos llama la atención que, con frecuencia en los procesos de entrenamiento, no siempre se tiene en cuenta la incidencia del ciclo menstrual en la planificación e individualización del entrenamiento deportivo; el no considerar lo anterior trae consigo que se rompa el principio de individualización y por consiguiente el rendimiento deportivo de las deportistas, puede verse afectado durante los días de la menstruación.

Algo muy preocupante y que nos hace coincidir con lo citado por Bataller (2017), es que, a lo largo del tiempo, los programas de entretenimiento han sido diseñados principalmente tomando en cuenta el sexo masculino, sin respetar las diferencias fisiológicas que puedan existir entre el hombre y la mujer. Esto como es lógico es negativo dentro de la preparación de las deportistas; precisamente por la falta de adaptabilidad de los programas de entrenamiento. Estamos de acuerdo en que, si se tuviera en cuenta el ciclo hormonal en la mujer, se conociera su funcionamiento y características individuales se pudiera adaptar el programa de entrenamiento y por ende incidir positivamente en su preparación y rendimiento deportivo.

Exclusivo interés tiene lo señalado en el caso de las nadadoras. Al ser considerado la natación como un deporte muy completo, el cual exige de una óptima condición física, cabe preguntarse ¿En qué medida incide el ciclo menstrual en el rendimiento deportivo de las nadadoras? Es por esto que el objetivo del estudio busca reflexionar sobre la incidencia del ciclo menstrual en la optimización del rendimiento deportivo de las nadadoras. 
El ciclo menstrual. Consideraciones generales sobre su incidencia en el rendimiento deportivo de las femeninas.

Rodriguez \& Curell (2017) definen el ciclo menstrual como el resultado de interacciones neuronales y endocrinas, mientras que Godoy \& Coll (2010) explican que se trata de un proceso producido por acciones precisas del sistema nervioso central, el ovario, la hipófisis y el sistema reproductor femenino.

Según autores precedentes el ciclo menstrual está conformado por cuatro fases, las cuales se denominan: folicular o post-menstrual, la ovulatoria, la luteínica o pre-menstrual y la menstrual o menstruación. Saldarriaga \& Artuz (2010) y Konovalova (2013) plantean que la duración del Ciclo Menstrual en la población en general es de 28 días, con posibles desviaciones de hasta siete días, pero en el caso de las deportista suele comportarse en una oscilación de 28 días en un $60 \%$ de los casos, mientras que en otros puede ser de 21 días e incluso llegar hasta 30-35 días, para un aproximado del $12 \%$.

La incidencia o no del ciclo menstrual en el rendimiento deportivo es un tema que además de ser muy controversial, ha venido tratándose en la literatura internacional desde hace muchos años. Las investigaciones realizadas por Guerra (1976); Díaz y cols. (1994); Gorostiaga (1996); Palacios Gil-Antuñano (2001) y más adelante Rello (2010) y otros autores demuestran la relación entre el ciclo menstrual y el rendimiento deportivo.

Desde 1963, los estudios realizados por Pierson y Lockhart, ayudan a entender el modo en que el organismo femenino responde ante la práctica deportiva. En este sentido Aguilar, Miranda, \& Quintana (2017), abordan la relación entre los efectos de la actividad física sobre la función reproductiva femenina y la influencia del ciclo menstrual en el rendimiento y optimización de las diferentes capacidades.

La autora coincide con Arias, et al. (2018), en que el ciclo menstrual es uno de los procesos biológicos más importantes de las mujeres, debido a la interacción de una serie de hormonas que dan lugar a diversos cambios tanto en el aspecto reproductivo de la mujer como en muchos otros tejidos del cuerpo. En este orden señala Seoane (2013), que el rendimiento deportivo en la mujer está diferenciado de los hombres no sólo por las diferencias biológicas, sino también por las diferencias psicológicas, sociológicas y culturales.

Se comparte con Prado (2013) en que para contribuir a un mejor proceso de preparación de las mujeres en las distintas modalidades deportivas y prevenir las posibles afecciones de la función menstrual, es importante considerar que la mujer a diferencia del hombre, debido a que está expuesta a continuos cambios hormonales derivados del ciclo menstrual su rendimiento es variable y como es lógico esto influye en sus resultados. Para Konovalova (2013), el estudio de la relación entre el ciclo menstrual y el rendimiento deportivo es un tema recurrente en las últimas investigaciones sobre la práctica deportiva en el sexo femenino. 
Konovalova (2013), enfatiza en que el conocimiento de la duración del ciclo y su estabilidad permite organizar la estructura del proceso de entrenamiento, de tal manera, que los mesociclos se programen acorde con los ciclos biológicos de la deportista, donde las fases más favorables (postmenstrual y postovulatoria) puedan asumir una carga mayor, alternándola con descensos en las fases de tensión fisiológica, ovulatoria, premenstrual y menstrual.

En su trabajo con deportistas vallecaucanas, Konovalova \& Rivera (2012), demostraron que las diferentes cualidades físicas tienen una dinámica variada a lo largo del ciclo, en el que las fases postmenstrual y postovulatoria se caracterizaron por el ascenso de la velocidad cíclica, la fuerza máxima, la fuerza explosiva y la resistencia, mientras que la flexibilidad tuvo los índices más altos en las fases menstrual y premenstrual.

Como puede apreciarse las teorías planteadas por Konovalova, en el 2013 y otros autores precedentes además de estar sustentadas en el principio de individualización del entrenamiento, refuerzan la idea en relación con la necesidad de respetar las características e individualidades en el proceso de planificación y desarrollo del entrenamiento deportivo; sobre todo cuando se está trabajando con deportistas que se encuentren en el ciclo menstrual.

Lo paradójico es que, aunque el tema relacionado con el ciclo menstrual y su incidencia en el rendimiento deportivo es de vital importancia, para los entrenadores, directivos y por supuesto para las deportistas, aun no se logra unificar criterios en relación a cuál es el nivel de incidencia de este en el rendimiento deportivo de las deportistas. Es importante considerar además que en el caso de Ecuador como en otros países, desafortunadamente los sistemas de desarrollo deportivo en ocasiones presentan falencias metodológicas que excluyen las características individuales de los deportistas, desconociendo las diferencias que existen entre hombres y mujeres e incluso las particularidades internas dentro del mismo género (Marroquín, 2017).

Esto crea una brecha teórico-práctica que requiere de urgente atención en nuestro contexto. Somos del criterio que para poder lograr la optimización del rendimiento deportivo es necesario tener en cuenta el principio de individualización y las características de cada deportista, considerando esencial dentro de esto su ciclo menstrual.

\section{¿Podrán las deportistas tener el mismo rendimiento deportivo durante el ciclo menstrual?}

Ante la diversidad de opiniones respecto al nivel de rendimiento que se puede lograr durante el ciclo menstrual en las deportistas, es oportuno considerar lo planteado por Platonov et al. (2012), el autor cita: que el deporte olímpico contemporáneo plantea altas exigencias para sus participantes, en particular a las mujeres. Durante la preparación y la participación en las competencias, las deportistas soportan cargas extremadamente altas, que pueden influir negativamente en su salud, en especial, en la función reproductiva, 
sistema cardiovascular, estado del tejido óseo, propiciando el desarrollo de distintas patologías.

Esta primera idea coincide con Konovalova (2013), al señalar que la ampliación del programa olímpico femenino propone no solamente la necesidad de un profundo estudio científico, sino también de la profilaxis de las patologías relacionadas con el ciclo menstrual, con el fin de conservar la función reproductiva de las mujeres deportistas

Una vez que se conocen estas notables diferencias en las deportistas, se da un paso importante para los entrenadores que buscan el desarrollo constante de su labor y así puedan alcanzar altos rendimientos con sus atletas, lo que les lleva a comprender mejor las aptitudes en la práctica diaria, seleccionar de una manera eficiente las tareas específicas y el trato para que contribuyan a una mejor individualización del entrenamiento, para mejorar el rendimiento y la calidad de vida de las mujeres deportistas.

Según el Doctor Burden (2019), Director Técnico de Fisiología en el Instituto Inglés del Deporte (EIS), "El ciclo menstrual se ha visto durante mucho tiempo como una barrera para el entrenamiento y el rendimiento", sin embargo enfatiza en que si este proceso fisiológico se observa desde otros puntos de vista puede ser usado como un verdadero potencial, ya que permite utilizar las fluctuaciones hormonales durante el entrenamiento, controlando desde cómo se mueve la mujer hasta cómo piensa durante su ciclo menstrual. Destaca que comprender que ocurre en estas fases permite a los entrenadores adaptar el entrenamiento y por ende optimizar los ejercicios y el resultado

Para la autora de esta investigación, es necesario situarnos en dos puntos fundamentales: la primera idea a tener en cuenta es que independientemente de las investigaciones realizadas las cuales han intentado demostrar que sí existe una estrecha relación entre el ciclo menstrual y el rendimiento deportivo, aún no se logra un criterio único al respecto, dando lugar a la segunda idea y es precisamente que en las investigaciones realizadas se reafirma la necesidad de profundizar en el diagnóstico y características de cada deportista para poder considerar dentro del proceso de planificación del entrenamiento deportivo la adaptabilidad de las actividades y acciones a desarrollar desde el principio de individualización del entrenamiento y de este modo potenciar el óptimo rendimiento deportivo.

Es por ello tan importante situarnos en la relación que se da entre el ciclo menstrual y los niveles de rendimiento deportivo de cada deportista y de esta forma valorar su incidencia de manera individualizada.

\section{El ciclo menstrual y su incidencia en el rendimiento deportivo de las nadadadoras.}

La natación es un deporte muy completo y divertido. Su práctica trae múltiples beneficios para el organismo, constituyendo un nuevo aprendizaje y un seguro de vida (Contreras, 2011). En el caso de la mujer su utilidad tiene un valor extraordinario, sin embargo, a 
pesar de ser un deporte ancestral y estar legalmente reconocido tanto a nivel internacional como nacional, no siempre es posible practicarlo; entre otros aspectos por la propia cultura, la carencia de recursos en cuanto a la accesibilidad a los lugares donde pueda practicarse o simplemente por limitaciones y perjuicios. Un ejemplo de lo anterior es precisamente los tabúes que existen acerca del ciclo menstrual y la práctica de la natación.

En el caso de las nadadoras es fundamental considerar los efectos del ciclo menstrual en la optimización del rendimiento deportivo. Entender la relación que se da entre el ciclo menstrual y el rendimiento deportivo, presupone según Konovalova (2013), tener en cuenta varios enfoques. Destaca la autora que se requeire profundizar en las particularidades de cada deportista en cuanto a su ciclo menstrual e ir evaluando las formas metodológicas más adecuadas para su entrenamiento.

Se coincide con Vrubliovskiy (2003) y Konovalova (2013), en que el entrenador necesita saber cuál es la duración del ciclo menstrual de sus deportistas, puesto que, de esta manera, se puede calcular el número de días "favorables" para la capacidad de trabajo durante el año y programar mejor el proceso de entrenamiento pág. (294).

Caballero \& Lafaurie (2019), destacan en las conclusiones de su investigación que la menstruación es una condición que afecta el bienestar y desempeño de las nadadoras de élite, por lo tanto, es necesario que sus implicaciones fisiológicas, psicológicas y sociales sean reconocidas en escenarios de alto rendimiento deportivo para brindar un mejor apoyo a estas atletas, lo que contribuirá a mejorar su calidad de vida y salud integral en general.

Lo manifestado permite asumir que en la natación al igual que en otros deportes practicado por las féminas se requiere tener en cuenta el ciclo menstrual para poder prever en la planificación del entrenamiento deportivo, la individualización del sistema de entrenamiento y actividades, lo cual conducirá a mejorar el rendimiento deportivo.

\section{Metodología.}

La investigación sigue una metodología descriptiva, no experimental con la utilización de métodos teóricos, tales como: el histórico-lógico, el analítico - sintético, inductivo deductivo y la revisión bibliográfica; todo ello permitió realizar un rastreo bibliográfico mediante la revisión y análisis de las diferentes bases y fuentes en un rango comprendido entre el 2010 y el 2020, obteniéndose de las bases consultadas como Scopus, PubMed, Google Académico, Scielo, Redalcy, Dialnet, Latindex, Web of Science, Publice información relevante sobre la incidencia del ciclo menstrual en el rendimiento deportivo. Para encontrar información adicional se realizó una búsqueda en los repositorios nacionales e internacionales que contemplan Tesis de Doctorado y Maestría.

Considerando la importancia de tener información confiable y de calidad, la estrategia de búsqueda seguida fue modificándose según cada base de datos consultada, identificando 
los artículos y restringiendo la población a consultar según las palabras claves: ciclo menstrual, natación, rendimiento deportivo.

\section{Criterios de inclusión}

1. Tipo de estudios: Estudios de revisiones teóricas o experimentales que sistematicen o desarrollen teorías en relación con el ciclo menstrual y su incidencia en el rendimiento deportivo de las deportistas femeninas, con especial interés en las nadadoras.

2. Tipo de participantes: deportistas femeninas practicantes de diferentes deportes

3. Tipo de resultados: Metodologías, modelos, programas que contemplen dentro del proceso de entrenamiento el ciclo menstrual para mejorar el rendimiento deportivo.

4. Tesis de Doctorado o Maestría realizadas en los 10 últimos años en universidades Internacionales o Nacionales sobre la influencia del ciclo menstrual en deportistas.

\section{Criterios de exclusión}

1. Estudios o investigaciones realizadas en un rango de tiempo diferente al contemplado en la investigación y que no aporten elementos significativos para nuestro estudio.

2. Artículo en idioma diferente del español o inglés.

3. Otros estudios que por su tema o resultados no aporten elementos significativos para nuestra investigación

\section{Evaluación de la calidad}

La calidad se determinó mediante el seguimiento riguroso de los criterios de inclusión y exclusión establecidos previamente.

\section{Análisis y discusión de resultados en cuanto a bases de datos consultadas:}

Según las bases de datos consultadas y considerando el rango de tiempo comprendido entre el 2010 y el 2020, se determinan como investigaciones potencialmente relevantes un total de 20 investigaciones, de las cuales, el 100\% maneja de forma combinada las variables: ciclo menstrual y rendimiento deportivo y 3 se dirigen a la incidencia del ciclo menstrual en nadadoras lo que representa el $15 \%$ de las fuentes consultadas. Se consultaron además 3 Tesis de Doctorado relacionadas con el tema, no encontrándose investigaciones suficientes en los repositorios tesis de maestría sobre este tema. Derivado de la revisión bibliográfica los resultaron evidencian que el año 2017, ha sido uno de los años con mayor producción científica relacionada con esta temática. 
Dentro de los autores que más aportaron al análisis realizado se destacan: Rello (2010 y 2015), Konovalova (2012 y 2013), Seoane (2013), Aguilar, Miranda, \& Quintana (2017), Arias, et al. (2018), y Caballero, \& Lafaurie (2020). Siendo de gran interés los estudios de Arias, et al. (2018), dentro de los autores ecuatorianos que han trabajado el tema, ya que son limitadas las investigaciones realizadas en Ecuador y que además cumplan con los criterios de inclusión determinados previamente.

La Tabla No1. Muestra un resumen de los resultados del análisis realizado en cuanto a las investigaciones potenciales directos para la presente investigación.

Tabla No 1 Resultado de Investigaciones potenciales directos para la investigación

\begin{tabular}{|c|c|c|c|}
\hline Autorías, Titulo y año & Base de Datos & Intervención- & Conclusiones \\
\hline $\begin{array}{lr}\text { Godoy, A., Guilarte, Y., } \\
\text { Hernández, P. }(2010) . \\
\text { Menstruación } & \text { y } \\
\text { rendimiento. } & \end{array}$ & $\begin{array}{l}\text { Educación Física y } \\
\text { Deportes, Revista Digital, } \\
\text { 14(140). http:// } \\
\text { www.efdeportes.com.Rev } \\
\text { ista Digital -Buenos Aires } \\
\text {-año 14-No } 140\end{array}$ & $\begin{array}{l}\text { La investigación busca } \\
\text { sistematizar las teorías en } \\
\text { relación con la influencia del } \\
\text { ciclo menstrual en el } \\
\text { rendimiento de } \\
\text { deportistas. analiza los } \\
\text { estudios precedentes }\end{array}$ & $\begin{array}{l}\text { La investigación muestra tres } \\
\text { ideas fundamentales: 1. El } \\
\text { control del ciclo menstrual es } \\
\text { una variable a considerar en la } \\
\text { planificación del entrenamiento, } \\
\text { para optimizar las fases del ciclo } \\
\text { donde es más oportuna la } \\
\text { elevación de las cargas de } \\
\text { trabajo. 2. Las fases del ciclo } \\
\text { menstrual de mayor } \\
\text { aprovechamiento son la post } \\
\text { menstrual y la post ovulatoria } \\
\text { por el aumento de la producción } \\
\text { de estrógenos y progesterona } \\
\text { hormonas determinantes en el } \\
\text { alto rendimiento femenino. } 3 \text {. } \\
\text { No existe criterio único por parte } \\
\text { de los diferentes autores, por lo } \\
\text { que la sistematización del } \\
\text { estudio de este importante tema } \\
\text { será piedra angular en el } \\
\text { mejoramiento de los resultados } \\
\text { deportivos de las mujeres }\end{array}$ \\
\hline $\begin{array}{l}\text { Rello, CF., (2010), } \\
\text { Interrogantes sobre la } \\
\text { actividad física y el ciclo } \\
\text { menstrual }\end{array}$ & $\begin{array}{l}\text { Educación Física y } \\
\text { Deportes, Revista Digital. } \\
\text { Buenos Aires, Año 15, No } \\
\text { 151, diciembre del } \\
\text { 2010.http:// } \\
\text { www.efedeportes.com/ }\end{array}$ & $\begin{array}{l}\text { Analiza los diferentes } \\
\text { posicionamientos asumidos } \\
\text { por autores precedentes en } \\
\text { torno a la incidencia del } \\
\text { ciclo menstrual en el } \\
\text { rendimiento deportivo de las } \\
\text { atletas }\end{array}$ & $\begin{array}{l}\text { Concluye con los aportes de los } \\
\text { diferentes autores y señala que } \\
\text { se trata de un tema muy } \\
\text { controversial debido a los } \\
\text { resultados derivados de las } \\
\text { diferentes investigaciones y de } \\
\text { la propia realidad práctica }\end{array}$ \\
\hline $\begin{array}{l}\text { Rello, CF., (2011), } \\
\text { Beneficios de la práctica } \\
\text { de la actividad física } \\
\text { durante la menstruación }\end{array}$ & $\begin{array}{l}\text { Educación Física y } \\
\text { Deportes, Revista Digital. } \\
\text { Buenos Aires, Año 15, No } \\
\text { 153.Fenrero 2011 } \\
\text { http:// } \\
\text { www.efedeportes.com/ }\end{array}$ & $\begin{array}{l}\text { Realiza una síntesis de los } \\
\text { beneficios que reporta el } \\
\text { ejercicio físico sobre los } \\
\text { efectos de la menstruación. }\end{array}$ & $\begin{array}{l}\text { Enfatiza en los beneficios de } \\
\text { orden fisiológico y psicológico, } \\
\text { resaltando que para poder } \\
\text { alcanzar los beneficios se } \\
\text { requiere de cumplir con } \\
\text { determinados parámetros como } \\
\text { es la dosificación de la } \\
\text { intensidad, duración y la } \\
\text { regularidad durante la práctica } \\
\text { deportiva. }\end{array}$ \\
\hline $\begin{array}{lrr}\text { Zanin, } & \text { L., } & \text { Paez, } \\
\text { Correa, C., y } & \text { De Bortoli, } \\
\text { M., } \quad(2011) . & \text { Ciclo } \\
\text { menstrual: sintomatología }\end{array}$ & $\begin{array}{l}\text { Fundamentos } \\
\text { Humanidades Universidad } \\
\text { Nacional de San Luis - } \\
\text { Argentina Año XII - }\end{array}$ & $\begin{array}{l}\text { Indaga sobre la distribución } \\
\text { de síntomas psicológicos y } \\
\text { físicos a lo largo del ciclo } \\
\text { menstrual, evaluar las }\end{array}$ & $\begin{array}{l}\text { Como conclusión infieren que } \\
\text { los síntomas inherentes al ciclo } \\
\text { no afectan el desempeño } \\
\text { habitual de las participantes por }\end{array}$ \\
\hline
\end{tabular}




\begin{tabular}{|c|c|c|c|}
\hline $\begin{array}{l}\text { y regularidad del estilo de } \\
\text { vida diario }\end{array}$ & $\begin{array}{l}\text { Número II } \quad(24 / 2011) \\
103 / 123 \text { pp. }\end{array}$ & $\begin{array}{lcr}\text { posibles } & \text { variaciones } & \text { del } \\
\text { estilo de vida diario y } & \text { su } \\
\text { relación con } & \text { la } \\
\text { sintomatología } & & \end{array}$ & $\begin{array}{l}\text { lo que serían síntomas propios } \\
\text { de la ciclicidad } \\
\text { femenina. }\end{array}$ \\
\hline $\begin{array}{l}\text { Seoane Prado A. (2013). } \\
\text { Influence of menstrual } \\
\text { cyclelin synchronized } \\
\text { swimming flexibility. }\end{array}$ & $\begin{array}{l}\text { AGON. International } \\
\text { Journal of Sport Sciences. } \\
\text { 3(2),!53M59 }\end{array}$ & $\begin{array}{l}\text { Investigar si existen } \\
\text { diferencias en el grado de } \\
\text { flexibilidad durante las } \\
\text { diferentes fases del ciclo } \\
\text { menstrual en } 10 \text { deportistas } \\
\text { de natación sincronizada, } \\
\text { con edades comprendidas } \\
\text { entre } 13 \text { y } 17 \text { años }\end{array}$ & $\begin{array}{l}\text { Concluyen que dada las } \\
\text { diferencias que se dieron en las } \\
\text { participantes el ciclo menstrual } \\
\text { afecta de forma significativa a la } \\
\text { flexibilidad pudiendo afectar al } \\
\text { rendimiento en natación } \\
\text { sincronizada. Lo cual tiene gran } \\
\text { importancia para el tema de } \\
\text { nuestra investigación. }\end{array}$ \\
\hline $\begin{array}{l}\text { Konovalova, E.,\& Rivera } \\
\text { M (2012), } \\
\text { Dinámica del rendimiento } \\
\text { de las jóvenes deportistas } \\
\text { durante el ciclo menstrual }\end{array}$ & $\begin{array}{l}\text { Programa } \\
\text { Universidad del Vditorial } \\
\text { 2012. } 120 \text { p.; } 24 \mathrm{~cm} .-- \\
\text { (Ciencias naturales y } \\
\text { exactas) }\end{array}$ & $\begin{array}{l}\text { El estudio evalúa la } \\
\text { dinámica de las cualidades } \\
\text { físicas de las jóvenes } \\
\text { deportistas en las diferentes } \\
\text { fases del ciclo menstrual y se } \\
\text { caracterizan las } \\
\text { particularidades de la } \\
\text { formación de la función } \\
\text { menstrual y su relación con } \\
\text { el rendimiento en las } \\
\text { jóvenes deportistas } \\
\text { vallecaucanas. }\end{array}$ & $\begin{array}{l}\text { Aporta la caracterización de } \\
\text { jóvenes } \\
\text { vallecaucanas en función de las } \\
\text { individualidades del ciclo } \\
\text { menstrual }\end{array}$ \\
\hline $\begin{array}{l}\text { Konovalova }(2013) \text { El } \\
\text { ciclo menstrual y el } \\
\text { entrenamiento deportivo: } \\
\text { Una mirada al problema }\end{array}$ & $\begin{array}{l}\text { Rev. U.D.C.A Act. \& Div. } \\
\text { Cient. 16(2): 293-302, } \\
\text { Julio-Diciembre, } 2013\end{array}$ & $\begin{array}{l}\text { Aplico un estudio con el fin } \\
\text { de relacionar las } \\
\text { características del ciclo } \\
\text { menstrual, su duración, } \\
\text { estabilidad, la fase de } \\
\text { menstruación, síntomas } \\
\text { menstruales }\end{array}$ & $\begin{array}{l}\text { Permitió corroborar el efecto } \\
\text { positivo de la práctica deportiva } \\
\text { durante el ciclo menstrual, } \\
\text { disminuyendo la presencia de } \\
\text { los síntomas premenstruales y } \\
\text { menstruales, al igual que el } \\
\text { mejoramiento del estado de } \\
\text { ánimo y estado físico en general. }\end{array}$ \\
\hline $\begin{array}{l}\text { Ramírez } \\
\text { Almudena. } \\
\text { Efectos de las fases del } \\
\text { ciclo menstrual sobre la } \\
\text { condición física, } \\
\text { parámetros fisiológicos y } \\
\text { psicológicos en mujeres } \\
\text { jóvenes moderadamente } \\
\text { entrenadas. }\end{array}$ & $\begin{array}{l}\text { Repositorio Universidad } \\
\text { de Extremadura. } \\
\text { Reppaña. } \\
\text { Repositorio } \\
\text { Doctoral }\end{array}$ & $\begin{array}{l}\text { Investigó sobre los efectos } \\
\text { de las fases del ciclo } \\
\text { menstrual en los aspectos } \\
\text { fisiológicos, psicológicos y } \\
\text { de condición física de la } \\
\text { mujer, para ello eligió una } \\
\text { muestra de } 32 \text { mujeres } \\
\text { jóvenes moderadamente } \\
\text { entrenadas, las cuales se } \\
\text { evaluaron durante las } \\
\text { diferentes fases del ciclo } \\
\text { menstrual (menstruación, } \\
\text { fase folicular y fase lútea) }\end{array}$ & $\begin{array}{l}\text { Concluyó que el ciclo menstrual } \\
\text { de la mujer moderadamente } \\
\text { entrenada no altera la cantidad } \\
\text { de actividad física y que sólo la } \\
\text { fase lútea podría repercutir en el } \\
\text { rendimiento físico }\end{array}$ \\
\hline $\begin{array}{l}\text { Marín, F., \& Herrera, AS } \\
\text { (2016) Variaciones en la } \\
\text { flexibilidad durante el } \\
\text { ciclo menstrual en } \\
\text { deportistas universitarias. }\end{array}$ & $\begin{array}{l}\text { Pensar en Movimiento: } \\
\text { Revista de Ciencias del } \\
\text { Ejercicio y la Salud, Vol. } \\
\text { 14, No. 2, Costa Rica. }\end{array}$ & $\begin{array}{l}\text { Investigan si } \text { existen } \\
\text { variaciones en la flexibilidad } \\
\text { en grupo de } 28 \text { mujeres } \\
\text { atletas universitarias, en } 2 \\
\text { fases del ciclo menstrual } \\
\text { (menstrual y ovulatoria). } \\
\text { Para ello se aplicaron } \\
\text { pruebas de flexibilidad } \\
\text { funcionales y multi- } \\
\text { articulares }\end{array}$ & $\begin{array}{l}\text { Destacan como conclusión que } \\
\text { no existieron variaciones } \\
\text { significativas en la flexibilidad a } \\
\text { lo largo del ciclo menstrual en la } \\
\text { población general. Sin embargo, } \\
\text { ciertas condiciones } \\
\text { sociodemográficas y clínicas } \\
\text { parecieran predisponer a } \\
\text { variaciones durante el mismo. }\end{array}$ \\
\hline $\begin{array}{l}\text { Jiménez Pérez, Y. (2016). } \\
\text { Las capacidades físicas } \\
\text { durante las etapas del ciclo }\end{array}$ & $\begin{array}{l}\text { Repositorio Universidad } \\
\text { Central "Marta Abreu" de } \\
\text { Las Villas. Facultad de } \\
\text { Cultura Física y el Deporte }\end{array}$ & \begin{tabular}{llr}
\multicolumn{3}{l}{ La investigación se dirigió a } \\
diseñar un & plan & de \\
actividades & para & el \\
desarrollo & de & las
\end{tabular} & $\begin{array}{l}\text { El estudio concluyo que las } \\
\text { capacidades físicas de fuerza, } \\
\text { resistencia y rapidez presentaron } \\
\text { cambios en las } 4 \text { etapas del ciclo }\end{array}$ \\
\hline
\end{tabular}




\begin{tabular}{|c|c|c|c|}
\hline $\begin{array}{l}\text { menstrual en atletas } \\
\text { juveniles de Tenis }\end{array}$ & $\begin{array}{lr}\text { Manuel } & \text { Fajardo. } \\
\text { Departamento } & \text { Didáctica } \\
\text { del Deporte. Cuba. Tesis } \\
\text { Doctoral }\end{array}$ & $\begin{array}{l}\text { capacidades físicas de las } \\
\text { mujeres tenistas durante las } \\
\text { etapas del ciclo menstrual. }\end{array}$ & $\begin{array}{l}\text { menstrual en la muestra. Aporta } \\
\text { datos para el diseño de un plan } \\
\text { de entrenamiento en el que se } \\
\text { tenga en cuenta las capacidades } \\
\text { de las mujeres durante etapas de } \\
\text { su ciclo menstrual. }\end{array}$ \\
\hline $\begin{array}{l}\text { Ríos Castelló, P. (2016). } \\
\text { Efecto de las fases del } \\
\text { ciclo menstrual sobre el } \\
\text { rendimiento aeróbico de } \\
\text { jóvenes triatletas. }\end{array}$ & $\begin{array}{l}\text { Repositorio } \begin{array}{r}\text { Universidad } \\
\text { de }\end{array} \text { Alicante. } \\
\text { Departamento r de } \\
\text { Didáctica General y } \\
\text { Didácticas Específicas } \\
\text { http://hdl. handle.n } \\
\text { et/10045/56288 } \\
\text { http://hdl.handle.net/1004 } \\
5 / 56288\end{array}$ & $\begin{array}{l}\text { Realizó una revisión } \\
\text { bibliográfica enfocada en el } \\
\text { rendimiento aeróbico } \\
\text { durante el ciclo menstrua, } \\
\text { unido a esto desarrolló una } \\
\text { propuesta para evaluar el } \\
\text { rendimiento deportivo en } \\
\text { deportista de triatlón }\end{array}$ & $\begin{array}{l}\text { Determina una contradicción } \\
\text { entre los resultados de la } \\
\text { propuesta al mostrar diferencias } \\
\text { en el rendimiento entre las } \\
\text { diferentes fases del ciclo } \\
\text { menstrual, lo cual se contradice } \\
\text { con el resultado encontrado en la } \\
\text { revisión bibliográfica parece } \\
\text { más clara la ausencia de } \\
\text { diferencia de rendimiento entre } \\
\text { fases" (Ríos Castelló, P., 2016, } \\
\text { p. 2) }\end{array}$ \\
\hline $\begin{array}{l}\text { Aguilar Macías Andrea } \\
\text { Selene;. Miranda Maria de } \\
\text { los Ángeles; Quintana } \\
\text { Díaz. Alfredo. La mujer, } \\
\text { el ciclo menstrual y la } \\
\text { actividad física (2017). }\end{array}$ & $\begin{array}{l}\text { Revista Archivo Médico } \\
\text { de Camagüey. } \\
\text { versión On- } \\
\text { line ISSN 1025-0255 } \\
\text { AMC vol.21 no.2 Camag } \\
\text { üey mar.-abr. } 2017\end{array}$ & $\begin{array}{l}\text { Describe } \\
\text { relacionados con la mujer, } \\
\text { su ciclo menstrual y la } \\
\text { práctica de actividad física. } \\
\text { Realizaron una revisión } \\
\text { bibliográfica de los últimos } \\
20 \text { años que incluyó } 53 \\
\text { artículos publicados en las } \\
\text { bases de datos biomédicas } \\
\text { (Scielo, Pubmed yedline), } \\
\text { así como repositorios de } \\
\text { tesis }\end{array}$ & $\begin{array}{l}\text { Indicaron que la relación entre } \\
\text { ciclo menstrual y actividad física } \\
\text { ha sido estudiada desde } \\
\text { diferentes perspectivas, } \\
\text { resultando significativo las } \\
\text { diferencias significativas en la } \\
\text { adaptación de la carga física en } \\
\text { diferentes fases del ciclo } \\
\text { menstrual. Recomiendan incluir } \\
\text { las variaciones del ciclo } \\
\text { menstrual en las planificaciones } \\
\text { de la actividad física femenina. }\end{array}$ \\
\hline 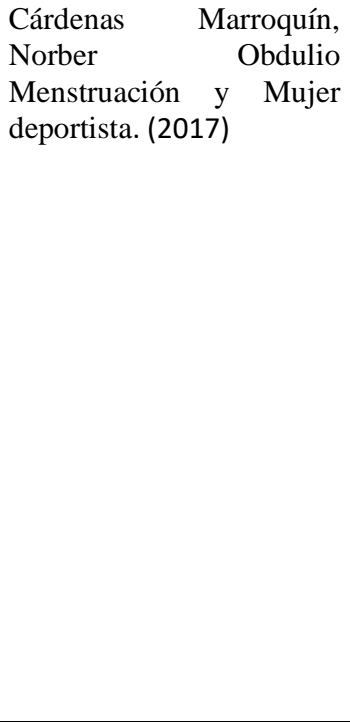 & $\begin{array}{l}\text { Revista digital: Actividad } \\
\text { Física y Deporte. }\end{array}$ & $\begin{array}{l}\text { Realizó la búsqueda de } \\
\text { información en Google } \\
\text { académico, documentos en } \\
\text { PDF de revistas digitales } \\
\text { como: Apunts, Cuadernos } \\
\text { de psicología y medicina del } \\
\text { deporte, entre otros. } \\
\text { Seleccionando los } \\
\text { documentos técnicos y } \\
\text { científicos, permitieran } \\
\text { obtener información sobre } \\
\text { temas relacionados con la } \\
\text { menarquia, el ciclo } \\
\text { menstrual en las mujeres } \\
\text { deportistas, considerando las } \\
\text { afecciones de la función } \\
\text { menstrual en las atletas y la } \\
\text { relación del rendimiento } \\
\text { deportivo con el ciclo } \\
\text { menstrual. }\end{array}$ & $\begin{array}{l}\text { Concluye que el conocimiento } \\
\text { de la duración del ciclo y su } \\
\text { estabilidad permite organizar la } \\
\text { estructura del proceso de } \\
\text { entrenamiento, de tal manera } \\
\text { que los mesociclos se programen } \\
\text { acorde con los ciclos biológicos } \\
\text { de la deportista, donde las fases } \\
\text { más favorables (postmenstrual y } \\
\text { postovulatoria) puedan asumir } \\
\text { una carga mayor, alternándola } \\
\text { con descensos en las fases de } \\
\text { tensión fisiológica (ovulatoria, } \\
\text { premenstrual y menstrual }\end{array}$ \\
\hline
\end{tabular}




\begin{tabular}{|c|c|c|c|}
\hline $\begin{array}{l}\text { Duaso Adriana, } \\
\text { Berzosa César , } \\
\text { Gutiérrez Héctor } \\
\text { Bataller, Ana Vanessa } \\
\text {, Campo Carlos Valero } \\
\text { (2017) Influencia del ciclo } \\
\text { menstrual en la fuerza } \\
\text { muscular: Una revisión } \\
\text { sistemática }\end{array}$ & $\begin{array}{l}\text { Revista Internacional de } \\
\text { Deportes Colectivos, } \mathrm{N}^{\circ} \text {. } \\
\text { 36, 2018, págs. } 48-52\end{array}$ & $\begin{array}{l}\text { El estudio contiene una } \\
\text { revisión sistemática entre los } \\
\text { años } 2007 \text { y } 2017 \text {, el cual } \\
\text { tuvo como objetivo } \\
\text { comprender las relaciones } \\
\text { entre las fluctuaciones } \\
\text { hormonales debidas al ciclo } \\
\text { menstrual y la fuerza } \\
\text { muscular que es capaz de } \\
\text { desarrollar una deportista. }\end{array}$ & $\begin{array}{l}\text { Los principales resultados } \\
\text { indican que aquellas fases del } \\
\text { ciclo asociadas a una mayor tasa } \\
\text { de producción de estrógenos } \\
\text { parecen estar relacionadas con } \\
\text { un aumento de la fuerza } \\
\text { muscular. Apunta además que la } \\
\text { influencia del ciclo menstrual } \\
\text { sobre aspectos fisiológicos que } \\
\text { pueden determinar el } \\
\text { rendimiento deportivo, } \\
\text { aseverando la necesidad de } \\
\text { profundizar en la investigación } \\
\text { de estas relaciones. }\end{array}$ \\
\hline $\begin{array}{l}\text { Wikstrom-Frisen, } 1 ., \\
\text { Boraxbekk, C. J., \& } \\
\text { Henriksson-larsen, K. } \\
\text { Effects on power, strength } \\
\text { and lean body mass of } \\
\text { menstrual/oral } \\
\text { contraceptive cycle based } \\
\text { resistance training. (2017) }\end{array}$ & 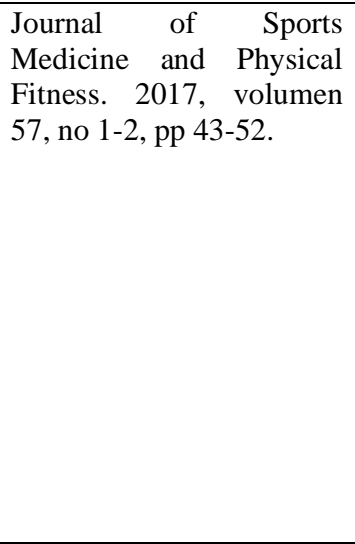 & $\begin{array}{l}\text { El objetivo del estudio se } \\
\text { centró en investigar los } \\
\text { efectos del ciclo } \\
\text { anticonceptivo menstrual y } \\
\text { oral en un entrenamiento de } \\
\text { resistencia de piernas } \\
\text { periodizado de } \begin{array}{rr}\text { alta } \\
\text { frecuencia en mujeres } \\
\text { entrenadas }\end{array}\end{array}$ & $\begin{array}{l}\text { Concluye que el entrenamiento } \\
\text { de resistencia de piernas } \\
\text { periodizado de alta frecuencia } \\
\text { durante las dos primeras } \\
\text { semanas del ciclo menstrual es } \\
\text { más beneficioso para optimizar } \\
\text { el entrenamiento que en las dos } \\
\text { últimas semanas. El } \\
\text { entrenamiento de resistencia } \\
\text { durante las dos primeras } \\
\text { semanas del ciclo menstrual } \\
\text { incluso resultó en una mayor } \\
\text { ganancia de masa corporal } \\
\text { magra que el entrenamiento } \\
\text { regular. }\end{array}$ \\
\hline $\begin{array}{l}\text { Figueroa Joaquín } \\
\text { Alchapar. (2017). } \\
\text { Ejercicio físico y función } \\
\text { reproductora en mujeres } \\
\text { deportistas de alto nivel }\end{array}$ & $\begin{array}{l}\text { Repositorio Universidad } \\
\text { Complutense de Madrid } \\
\text { Facultad de Medicina } \\
\text { Departamento r de } \\
\text { Fisiología Humana. Tesis } \\
\text { Doctoral. }\end{array}$ & $\begin{array}{l}\text { La investigación profundiza } \\
\text { en las relaciones existentes } \\
\text { entre el deporte de alto nivel } \\
\text { y la incidencia de } \\
\text { irregularidades menstruales, } \\
\text { Determinando la incidencia } \\
\text { de alteraciones menstruales } \\
\text { en } 4 \text { grupos de mujeres } \\
\text { deportistas de élite (NAD, } \\
\text { nadadoras; PIR, piragüistas; } \\
\text { TRI, triatletas; AT, atletas) }\end{array}$ & $\begin{array}{l}\text { Concluye que en mujeres } \\
\text { deportistas de élite se producen } \\
\text { alteraciones menstruales, con } \\
\text { diferente incidencia en función } \\
\text { del grupo deportivo } \\
\text { considerado. Destaca para } \\
\text { nuestra investigación los } \\
\text { resultados derivados de la } \\
\text { experiencia con nadadoras }\end{array}$ \\
\hline $\begin{array}{l}\text { Bustos, R., Arriagada, V,. } \\
\text { Briceño, K., Matamala, } \\
\text { Y., Pozo, P., Merino, F., } \\
\text { Brito,J., Parra, J., (2018), } \\
\text { Influencia de la Actividad } \\
\text { Física en r la } \\
\text { Sintomatología r del } \\
\text { Síndrome Premenstrual en } \\
\text { universitarias }\end{array}$ & $\begin{array}{l}\text { Revista chilena de } \\
\text { obstetricia y ginecología. } \\
\text { vol.83 no.1 Santiago feb. } \\
2018 \text {. } \\
\text { http://dx.doi.org/10.4067/ } \\
\text { s0717- } \\
75262018000100045\end{array}$ & $\begin{array}{l}\text { Los autores realizaron un } \\
\text { estudio observacional, } \\
\text { descriptivo, transversal, con } \\
\text { una muestra de } 340 \text { mujeres } \\
\text { escogidas al azar entre } 18 \text { a } \\
27 \text { años, estudiantes de la } \\
\text { Facultad de Medicina de la } \\
\text { Universidad de Concepción, } \\
\text { a las cuales se aplicaron } \\
\text { diferentes instrumentos para } \\
\text { el diagnóstico del trastorno } \\
\text { disfórico premenstrual y } \\
\text { para medir la intensidad del } \\
\text { dolor }\end{array}$ & $\begin{array}{l}\text { El estudio plantea como } \\
\text { conclusión que la actividad } \\
\text { física no tiene influencia sobre la } \\
\text { disminución de la } \\
\text { sintomatología del síndrome } \\
\text { premenstrual en la población } \\
\text { estudiada. Al cruzar las } \\
\text { variables de actividad física y la } \\
\text { sintomatología del síndrome } \\
\text { premenstrual se estableció que } \\
\text { no existía relación entre ellas } \\
\text { (valor } p=0,605) \text {. }\end{array}$ \\
\hline $\begin{array}{l}\text { Arias Moreno, E. R.; } \\
\text { Martínez Benítez, J. E.; } \\
\text { Goyes Acaro, F. O.; V } \\
\text { Ortiz Aldea, V. F.; } \\
\text { Montero, S. (2018). } \\
\text { Variabilidad en el } \\
\text { rendimiento físico de las } \\
\text { jugadoras de fútbol según } \\
\text { las fases del ciclo } \\
\text { menstrual. }\end{array}$ & 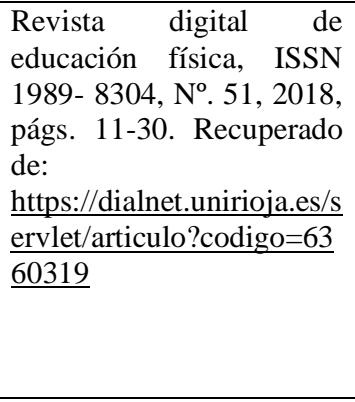 & $\begin{array}{l}\text { El objetivo del estudio buscó } \\
\text { determinar la influencia de } \\
\text { las fases del ciclo menstrual } \\
\text { en las diferentes fases del } \\
\text { ciclo menstrual (Fase } \\
\text { Menstrual-FM, Fase } \\
\text { Folicular-FF y Fase Lútea- } \\
\text { FL). En el rendimiento físico } \\
\text { de las jugadoras de fútbol de } \\
\text { la Universidad Autónoma de } \\
\text { Madrid (UAM). }\end{array}$ & $\begin{array}{l}\text { Como parte de las conclusiones } \\
\text { se destaca que en muchas } \\
\text { ocasiones los entrenadores } \\
\text { realizan sus planificaciones de } \\
\text { entrenamiento sin tener en } \\
\text { cuenta las necesidades } \\
\text { fisiológicas en mujeres } \\
\text { deportistas, dejando a un lado las } \\
\text { características que el ciclo } \\
\text { menstrual puede presentar en } \\
\text { cada fase. Concluye, que el }\end{array}$ \\
\hline
\end{tabular}




\begin{tabular}{|c|c|c|c|}
\hline & & & $\begin{array}{l}\text { rendimiento físico de las } \\
\text { deportistas estudiadas no se } \\
\text { encontró influenciado por las } \\
\text { diferentes fases del ciclo } \\
\text { menstrual. }\end{array}$ \\
\hline $\begin{array}{l}\text { Arango Barrientos Andrés } \\
\text { Felipe, Castaño Mejía } \\
\text { Edna Ximena, Gallo } \\
\text { Buitrago, Cristian Camilo, } \\
\text { Otalvaro Juan Pablo } \\
\text { Orozco.(2019), } \\
\text { Entrenamiento Físico y } \\
\text { Ciclo Menstrual }\end{array}$ & $\begin{array}{l}\text { Repositorio Universidad } \\
\text { Católica de Oriente. } \\
\text { Facultad de Educación. } \\
\text { Trabajo de Grado. } \\
\text { Rionegro- Antioquia }\end{array}$ & $\begin{array}{l}\text { Busca establecer mediante la } \\
\text { revisión bibliográfica el } \\
\text { reconocimiento y la } \\
\text { comprensión de las } \\
\text { relaciones existentes entre } \\
\text { ciclo menstrual y la } \\
\text { actividad física, }\end{array}$ & $\begin{array}{l}\text { Como parte de las conclusiones } \\
\text { de este estudio se infiere la } \\
\text { variabilidad que se da en cuanto } \\
\text { a rendimiento deportivo en } \\
\text { correspondencia con los días del } \\
\text { ciclo menstrual y el tipo de } \\
\text { actividad física planificada. }\end{array}$ \\
\hline $\begin{array}{l}\text { Caballero, A \& } \\
\text { Lafaurie, MM., (2020) } \\
\text { Nadar la con } \\
\text { menstruación: un estudio } \\
\text { cualitativo en nadadoras } \\
\text { de élite }\end{array}$ & $\begin{array}{l}\text { Revista de la Facultad de } \\
\text { Medicina Universidad } \\
\text { Nacional de Colombia }\end{array}$ & $\begin{array}{l}\text { El objetivo de la } \\
\text { investigación se dirigió a } \\
\text { describir la experiencia de } \\
\text { nadadoras de élite de una } \\
\text { liga deportiva de Bogotá } \\
\text { D.C. en relación con la } \\
\text { menstruación y su práctica } \\
\text { deportiva. }\end{array}$ & $\begin{array}{l}\text { La menstruación es una } \\
\text { condición que afecta el bienestar } \\
\text { y desempeño de las nadadoras } \\
\text { de élite, por lo tanto, es } \\
\text { necesario que sus implicaciones } \\
\text { fisiológicas, psicológicas y } \\
\text { sociales sean reconocidas en } \\
\text { escenarios de alto rendimiento } \\
\text { deportivo para brindar un mejor } \\
\text { apoyo a estas atletas, lo que } \\
\text { contribuirá a mejorar su calidad } \\
\text { de vida y salud integral en } \\
\text { general. }\end{array}$ \\
\hline
\end{tabular}

\section{Discusión de resultados}

Si bien es cierto que desde hace muchos años se ha venido tratando en la literatura el tema de la relación que existe entre ciclo menstrual y el rendimiento deportivo de las féminas en los diferentes deportes, también es necesario reflexionar en que este aspecto requiere una valoración más profunda e integral; si tenemos en cuenta que estamos ante un tema muy controversial: por una parte se encuentran las teorías presentadas por autores como: Guerra (1976); Díaz y cols. (1994); Palacios Gil-Antuñano (2001), Rello (2010 y 2015), Konovalova (2013 y 2015), Seoane (2013), Aguilar, Miranda, \& Quintana (2017), Arias y coll. (2018), entre otros, que sistematizan la relación entre ciclo menstrual y el rendimiento deportivo visto desde la perspectiva integral, en función de precisar las formas y metodologías del entrenamiento basadas en la individualización, adaptabilidad, variabilidad acorde con las características individuales de las deportistas para poder alcanzar un óptimo rendimiento deportivo; mientras que por otra parte también se reconocen los aportes de Bustos, Arriagada, Briceño, Matamala, Pozo, Merino, Brito, Parra (2018), los cuales indican que la actividad física no tiene influencia sobre la disminución de la sintomatología del síndrome premenstrual en poblaciones estudiadas.

Los autores mencionados en su investigación, al cruzar las variables de actividad física y la sintomatología del síndrome premenstrual establecen que no existía relación entre ellas (valor $\mathrm{p}=0,605$ ). En este orden llama la atención los estudios de Castelló (2016), al evaluar el efecto de las fases del ciclo menstrual sobre el rendimiento aeróbico de jóvenes triatletas; este estudio determina una contradicción entre los resultados de la propuesta al 
mostrar diferencias en el rendimiento entre las diferentes fases del ciclo menstrual, lo cual se contradice con el resultado encontrado en las revisiones bibliográficas, señala Castelló (2016, P. 2), que parece más clara la ausencia de diferencia del rendimiento entre fases".

Especial interés tiene también considerar en este análisis los resultados obtenidos por atletas de diferentes deportes en las competencias, las cuales estando en su ciclo menstrual en la fase de competición han alcanzado resultados sobresalientes, llegando incluso a obtener medallas olímpicas. Lo anterior demuestra algo que a criterio de la autora es fundamental tener en cuenta y está relacionado precisamente con teorías precedentes en relación con la importancia de la etapa de entrenamiento considerando la preparación e individualización del entrenamiento y el conocimiento detallado de las características e individualidades de las deportistas como única vía para garantizar el éxito (Konovalova, 2012 y 2013).

En esta línea la investigación realizada por: Wikstrom-Frisen, Boraxbekk, \& Henrikssonlarsen, en el (2017), sobre Effects on power, strength and lean body mass of menstrual/oral contraceptive cycle based resistance training, indican que el entrenamiento de resistencia de piernas periodizado de alta frecuencia durante las dos primeras semanas del ciclo menstrual señala que esta fase es más beneficiosa para optimizar el entrenamiento que en las dos últimas semanas. Señalando que el entrenamiento de resistencia durante las dos primeras semanas del ciclo menstrual incluso resultó en una mayor ganancia de masa corporal magra que el entrenamiento regular. Destacan que en mujeres deportistas de élite se producen alteraciones menstruales, con diferente incidencia en función del grupo deportivo considerado.

Otro de los estudios que guardan relación con el análisis es el realizado por Duaso, Gutiérrez, Bataller, Campo (2017), sobre la Influencia del ciclo menstrual en la fuerza muscular: los autores desde la revisión sistemática que desarrollan, indican que aquellas fases del ciclo asociadas a una mayor tasa de producción de estrógenos parecen estar relacionadas con un aumento de la fuerza muscular. Apunta además que la influencia del ciclo menstrual sobre aspectos fisiológicos que pueden determinar el rendimiento deportivo, aseverando la necesidad de profundizar en la investigación de estas relaciones.

Singular importancia para esta investigación, tiene los resultados del estudio desarrollado por: Konovalova (2012 y 2013), Marroquín (2017), Arias et al. (2018), los cuales en su generalidad indica que el conocimiento de la duración del ciclo su estabilidad permite organizar la estructura del proceso de entrenamiento, de tal manera que los mesociclos se programen acorde con los ciclos biológicos de la deportista, donde las fases más favorables (postmenstrual y postovulatoria) puedan asumir una carga mayor, alternándola con descensos en las fases de tensión fisiológica (ovulatoria, premenstrual y menstrual). Los estudios de Figueroa (2017), profundizan y comparten en las relaciones existentes entre el deporte de alto nivel y la incidencia de irregularidades menstruales, concluye que 
en mujeres deportistas de élite se producen alteraciones menstruales, con diferente incidencia en función del grupo deportivo considerado.

Destaca para nuestra investigación los resultados derivados de la experiencia con nadadoras y los aportes Konovalova (2013), al señalar que la teoría contemporánea del entrenamiento deportivo no contiene suficientes conocimientos para una racional planificación del entrenamiento de las mujeres; se conoce que en muchas ocasiones la planificación y planes de entrenamiento se realizan sobre la generalidades los atletas participantes, dejando de contemplar las particularidades individuales del organismo femenino, plantea la autora que los resultados de las investigaciones sobre la influencia de la fase menstrual en el resultado deportivo han sido variados, pero la mayoría de ellos apunta a que las deportistas consiguen sus mejores marcas en el periodo fuera de la menstruación.

Considerando y reflexionado sobre los aportes precedentes resulta básico resumir algunas ideas generales que forman parte concluyente del análisis realizado:

- Se coincide con Konovalova (2013), en que los resultados de las investigaciones precursoras sobre la influencia o no del ciclo menstrual en el rendimiento y resultado deportivo, han tenido opiniones muy variadas, pero la mayoría de ellos respaldan que las deportistas consiguen sus mejores marcas en el periodo fuera de la menstruación.

- Actualmente resulta fundamental antes de desarrollar los planes de entrenamiento valorar las características individuales y el diagnóstico asertivo de cada atleta, esto conducirá a profundizar en el nivel de conocimiento que se tenga de ellas y por consiguiente la planificación del entrenamiento puede realizarse de manera más personalizada, considerando siempre las fases de su ciclo menstrual.

- Es necesario considerar que existen diferencias significativas entre el sexo femenino y el masculino, entre un deporte y otro, así como de una atleta a otra, de ahí que la adaptación de la carga física en las diferentes fases del ciclo menstrual, debe incluir las variaciones individuales que pueden darse como consecuencia del ciclo menstrual, resultando fundamental tener este aspecto en cuenta en las planificaciones y procesos de entrenamiento a realizar (Aguilar, Miranda, \& Quintana, 2017, pp. 294-307).

- Entender la preparación del entrenamiento como un proceso primordial antes de las competencias implica considerar el ciclo menstrual, las variaciones individuales, el conocimiento y comunicación con las deportistas, como elementos esenciales para garantizar el éxito deportivo.

- En el caso de la natación es extremadamente importante considerar el principio de individualización del entrenamiento, ya que solo así se podrá garantizar el éxito deportivo en cuanto a rendimiento y resultados se refiere (Caballero \& Lafaurie, 2020). 
- Dada las limitaciones en el contexto ecuatoriano en cuanto a estudios relacionados con este tema se requiere direccionar la apertura de nuevas investigaciones en esta línea.

- Todo lo anterior nos permite asumir la necesidad de introducir cambios en los modelos y metodologías actuales de entrenamiento, los cuales deben basarse en el principio de la individualización y en el conocimiento de las deportistas en los diferentes deportes con especial interés en las nadadoras.

En resumen, en el contexto de la natación como en otros deportes, se trata de un tema altamente atrayente e importante a considerar como parte del entrenamiento deportivo; su pertinencia y actualidad hacen que sea necesario continuar profundizando en el mismo desde una visión integral del problema objeto de estudio.

A juicio de la autora no sólo es determinar si el ciclo menstrual incide o no, en el rendimiento deportivo, lo realmente importante es saber planificar y dosificar las cargas del entrenamiento en correspondencia con el nivel de potencialidades existente en cada nadadora, teniendo en cuenta en qué fase del ciclo menstrual la nadadora puede rendir mejor y qué hacer para alcanzar progresivamente los resultados esperados. Es saber respetar su ciclo biológico, donde las fases más favorables (postmenstrual y postovulatoria) puedan asumir una carga mayor, alternándola con descensos en las fases de tensión fisiológica (ovulatoria, premenstrual y menstrual). De ahí que se comparta con Konovalova (2013), y otros autores en que los ajustes en la magnitud de la carga en las distintas fases del ciclo, se deben hacer, en lo posible, acorde con las características del ciclo menstrual de cada deportista y de esta forma incidir en el rendimiento y resultados deportivos.

\section{Conclusiones}

A modo de conclusión podemos expresar que:

- Las tendencias en las teorías revisadas a pesar de la diversidad y controversia existente apuntan a establecer la relación entre el ciclo menstrual y el rendimiento deportivo de las atletas, con especial interés en las nadadoras; sin embargo debido a la variabilidad de criterios lo fundamental es continuar profundizando en esta línea de investigación, sobre todo en el contexto ecuatoriano, con el objetivo de poder adaptar y seleccionar los planes de entrenamiento a las características e individualidades de cada deportista.

- En correspondencia con lo señalado se considera que sí incide el ciclo menstrual en el rendimiento deportivo de las deportistas. Esto hace asumir posiciones teóricasprácticas que respaldan la necesidad de comprender la importancia de establecer un equilibrio entre las potencialidades de las deportistas durante su ciclo menstrual y las metodologías y exigencias a cumplimentar durante el entrenamiento deportivo. 
- El éxito en el rendimiento y resultado deportivo de cada atleta va a depender en gran medida del nivel de conocimientos y comunicación que se tenga con las nadadoras en la etapa previa a la competencia, ya que esto es lo que permite estructurar el proceso de entrenamiento y programar las actividades a realizar acorde con las fases más favorables dentro del ciclo menstrual

\section{Referencias Bibliográficas}

Arango, A., Castaño, E., Gallo, C, y Otalvaro, J. (2019). Entrenamiento Físico y Ciclo Menstrual (Tesis de Grado). Repositorio Universidad Católica de Oriente. Facultad de Educación. Rionegro- Antioquia, Colombia.

Arias, E., Martínez, J., Goyes, F., Ortiz, V. \& Montero, S. (2018). Variabilidad en el rendimiento físico de las jugadoras de fútbol según las fases del ciclo menstrual. Revista digital de educación física, 51, 11-30. Recuperado de: https://dialnet.unirioja.es/servlet/articulo?codigo $=6360319$.

Aguilar, AS \& Coll (2017), La mujer, el ciclo menstrual y la actividad física. Revista Archivo Médico de Camagüey 21 (2). Recuperado de https://bit.ly/39kfdr0

Allen, B. \& Miller K. (2019). El desarrollo físico en las niñas: qué esperar durante la pubertad. American Academy of Pediatrics FAAP.

Bataller, A. G. (2017). Estructura de microciclos de entrenamiento en función del ciclo menstrual. Comunicaciones técnicas, (4), 4-8. Recuperado de https://bit.ly/37c4a0j

Bentacourt, J. C., Sánchez, B., Arias, E., \& Barroso, E. (2020). Patrón de lateralidad en jugadores masculinos de baloncesto, reservas escolares y juveniles de La Habana. PODIUM-Revista de Ciencia y Tecnología en la Cultura Física, 15(3), 449459.

Burden, R,. Entrevista para la BBC (2019). Influencia del ciclo menstrual en los deportistas.

Bustos, R., Arriagada, V,. Briceño, K., Matamala, Y., Pozo, P., Merino, F., Brito,J. y Parra, J., (2018), Influencia de la Actividad Física en la Sintomatología del Síndrome Premenstrual en universitarias Revista chilena de obstetricia y ginecología 83 (1). http://dx.doi.org/10.4067/s0717-75262018000100045 .

Caballero, A \& Lafaurie, M M,. (2020), Swimming and menstruation: a qualitative study in elite female swimmers. Rev. Fac. Med, 68 (3) 356-62. Recuperado de http://dx.doi.org/10.15446/revfacmed.v68n3.76135.

Contreras C., (2011). La importancia de la práctica de la natación en la educación de los niños y las niñas. Lecturas: Educación Física y Deportes, Revista Digital, (161). Recuperado de http://www.efdeportes.com/efd161.

Díaz, B., y cols. (1994). Alteraciones endocrinas en la mujer deportista. Introducción a la medicina y ciencias del deporte (pp. 113-121). Servicio de publicaciones. Universidad de Oviedo. 
Duaso, A,. Berzosa, C,. Gutiérrez, H,. Bataller, AN,. Campo, CV,. (2017) Influencia del ciclo menstrual en la fuerza muscular: Una revisión sistemática. Revista Internacional de Deportes Colectivos, (36), 48-52. Recuperado de http://dx.doi.org/10.13140 / RG.2.2.34750.38727

Figueroa, J., (2017). Ejercicio físico y función reproductora en mujeres deportistas de alto nivel (Tesis Doctoral). Universidad Complutense de Madrid Facultad de Medicina Departamento de Fisiología Humana.

Guerra, T. (1976). Anovulatorios y deporte. Apuntes de Medicina Deportiva, 13 (50), 8388.

Godoy, L. A., Guilarte, Y., Hernández, P., \& Bonilla, J. L. (2010). Menstruación y rendimiento. Revista Digital EF Deportes, 140, 14. Recuperado de https://bit.ly/33kJ7Yg

Gorostiaga, E. (1996). Deporte y salud. Gobierno de Navarra, Departamento de Bienestar Social, Deporte y Juventud.

Jiménez Pérez, Y. (2016). Las capacidades físicas durante las etapas del ciclo menstrual en atletas juveniles de Tenis (Tesis Doctoral). Universidad Central "Marta Abreu" de Las Villas Facultad de Cultura Física y el Deporte Manuel Fajardo. Departamento Didáctica del Deporte, Cuba.

Konovalova, E. (2012). Caracterización de la capacidad de trabajo de las deportistas colombianas durante el ciclo menstrual. Sportyvna Meditsina (Ucrania), 1, 76-83. Recuperado de https://bit.ly/33nyR1E

Konovalova, E., \& Rivera Echeverry, M. (2012). Dinámica del rendimiento de las jóvenes deportistas durante el ciclo menstrual, Colombia: UNIVALLE.

Konovalova, E. (2013). El ciclo menstrual y el entrenamiento deportivo: una mirada al problema. Revista UDCA Actualidad \& Divulgación Científica, 16(2), 293-302. Recuperado de https://doi.org/10.31910/rudca.v16.n2.2013.900

Marroquín, N. O. C. (2017). Menstruación y mujer deportista. Revista digital: Actividad Física y Deporte, 3(1). Recuperado de https://bit.ly/2J1ZulR

Marín, F. M., \& Herrera, A. S. (2016). Variaciones en la flexibilidad durante el ciclo menstrual en deportistas universitarias. PENSAR EN MOVIMIENTO: Revista de Ciencias del Ejercicio y la Salud, 14(2), 1-14. Recuperado de http://dx.doi.org/10.15517/pensarmov.v14i2.23662

Ramírez, A,. (2015). Efectos de las fases del ciclo menstrual sobre la condición física, parámetros fisiológicos y psicológicos en mujeres jóvenes moderadamente entrenadas (Tesis doctoral). Universidad Extremadura, España.

Rello, CF. (2010a). Actividad física y menstruación: Análisis histórico. Revista Digital: Lecturas de Educación Física y Deportes, 149. Recuperado de https://bit.ly/2KKuxTF

Rello, CF., (2010b). Interrogantes sobre la actividad física y el ciclo menstrual. Revista Digital: Lecturas de Educación Física y Deportes, 151. Recuperado de https://bit.ly/378E6TX 
Rello, CF., (2011c), Beneficios de la práctica de la actividad física durante la menstruación. Revista Digital: Lecturas de Educación Física y Deportes, 153. Recuperado de https://bit.ly/37a1osC

Ríos Castelló, P. (2016). Efecto de las fases del ciclo menstrual sobre el rendimiento aeróbico de jóvenes triatletas (Tesis de grado). Repositorio Universidad de Alicante. Departamento de Didáctica General y Didácticas Específicas.

Jiménez, M. R., \& Aguilá, N. C. (2017). El ciclo menstrual y sus alteraciones. PediatríaIntegral, 304.

Palacios Gil-Antuñano, N. (2001). Ciclo menstrual y contenido mineral óseo en mujeres deportistas de élite. En Actas del VIII Congreso de la Federación Española de Medicina del Deporte y III Congreso Hispano-Luso de medicina del deporte. (pp. 4449). Diputación General de Aragón, Departamento de Cultura y Turismo.

Piaget, J., \& Inhelder, B. (1948). La repretation de I espace chez I enfant. Paris: PUF.

Platonov, V.N.; Bulatova, M.M.; Kosminina, E.S. (2012). Argumentos médicobiológicos para redireccionar el desarrollo de la parte femenina del programa de los Juegos Olímpicos. Sportyvna Meditsina, 1, 3-9. Recuperado de https://bit.ly/33mDOrv

Saldarriaga, W.; Artuz, M.A. (2010). Fundamentos de ginecología y obstetricia. Progr. Ed. Universidad del Valle, 69-129.

Sánchez, B., Lastres, A., Arias, E. R., Mesa, M., Vidarrueta, R., \& García, L. D. (2020). Deportes de combate, hacia un modelo de finalidad táctica de selección de talentos. PODIUM-Revista de Ciencia y Tecnología en la Cultura Física, 15(3).

Seoane, A., (2013). Influence of menstrual cyclelin synchronized swimming flexibility AGON. International Journal of Sport Sciences. 3(2), 53- 59. Recuperado de https://bit.ly/3mbMgkz

Vrubliovskiy, E. (2003). Dirección del proceso de entrenamiento de las mujeres en las modalidades atléticas de velocidad y fuerza. Teoría y práctica Cult. Fís, 6, 2-5.

Wikström-Frisén, L., Boraxbekk, C. J., \& Henriksson-Larsen, K. (2017). Effects on power, strength and lean body mass of menstrual/oral contraceptive cycle based resistance training. Journal of Sports Medicine and Physical Fitness, 57(1-2), 43-52. Recuperado de http://dx.doi.org/43-52.10.23736/S0022-4707.16.05848-5.

Zanin, L., Paez, A., Correa, C., \& De Bortoli, M. (2011). Ciclo menstrual: sintomatología y regularidad del estilo de vida diario. Fundamentos en Humanidades, 12(24), 103123. Recuperado de https://bit.ly/2V2JD8O

\section{LC Ciencia}




\section{PARA CITAR EL ARTÍCULO INDEXADO.}

Iturralde Guerra, L. Y. (2021). Influencia del ciclo menstrual en nadadoras. Ciencia Digital, 5(1), 73-92. https://doi.org/10.33262/cienciadigital.v5i1.1464

\section{Ciencia \\ LDigital}

El artículo que se publica es de exclusiva responsabilidad de los autores y no necesariamente reflejan el pensamiento de la Revista Ciencia Digital.

El artículo queda en propiedad de la revista y, por tanto, su publicación parcial y/o total en otro medio tiene que ser autorizado por el director de la Revista Ciencia

\section{Digital.}
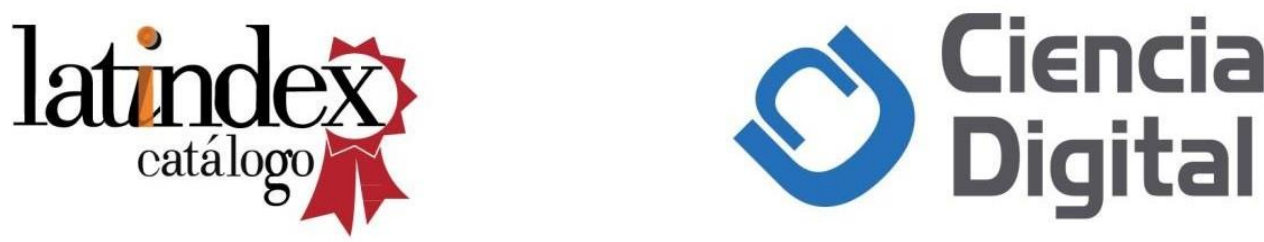